that found during the control test. We have shown, however, that despite these similar AVP concentrations at peak diuresis there was grossly impaired diuresis in 10 of the 12 subjects.

\section{Discussion}

We found that there was impaired water handling in most subjects given therapeutic doses of carbamazepine. This is therefore a physiological effect of the drug and not dependent on idiosyncrasy or additional drug treatment. The drug's effect on water metabolism may be shown only by stressing the subjects with a water load and in standard conditions is not enough to alter the plasma electrolyte concentrations. Only a minority of patients would be expected to develop overt water intoxication.

Our results do not suggest that the mode of action is by stimulating the secretion of antidiuretic hormone. The basal plasma AVP concentrations fell while subjects took the drug, which agrees with the results of other studies." 6 We found no change in the plasma osmolality or electrolyte concentrations, which suggests that there is a fundamental resetting of osmoreceptors so that during periods of dehydration less antidiuretic hormone is secreted for a given plasma osmolality.

While diuresis is impaired during carbamazepine treatment, the plasma AVP concentration at peak diuresis is not altered when compared with that during a control water load test. This may be taken as further evidence against direct stimulation of the secretion of antidiuretic hormone. It suggests, however, that there is increased renal sensitivity to "normal" plasma concentrations of AVP. If the feedback mechanism via the osmoreceptors is intact then antidiuretic hormone secretion will be further reduced, which will compensate for the increased renal sensitivity. Therefore, if increased renal sensitivity to antidiuretic hormone is postulated there must also be a resetting of osmoreceptor regulation. This implies that when there is a large water load the osmoreceptors direct the hypothalamus to secrete an inappropriately high level of antidiuretic hormone. This argument assumes that the plasma AVP concentration at peak diuresis is not lower during carbamazepine medication simply because AVP secretion cannot be switched off any more.

There have been two reports of patients with symptomatic water intoxication due to carbamazepine, whose plasma AVP concentrations were inappropriately high yet returned to normal when the drug dosage was reduced. ${ }^{*} \times$ This would agree with disturbed autoregulation such as we postulate.

Our results suggest that the antidiuretic action of carbamazepine is due to a combination of resetting of osmoreceptors and increased renal sensitivity to antidiuretic hormone. The response of the osmoreceptors is blunted, so that during dehydration too little antidiuretic hormone is secreted and during overhydration too much is secreted. Under the influence of carbamazepine the osmoreceptors become lazy.

\section{References}

Stephens, W P, et al, British Medical fournal, 1977, 1, 754.

2 Henry, D A, et al, British Medical fournal, 1977, 1, 83.

${ }^{3}$ Helin, I, et al, British Medical fournal, 1977, 2, 558.

4 Baylis, P H, and Heath, D A, Clinical Endocrinology, 1977, 7, 91.

5 Meinders, A E, Cejka, V, and Robertson, G L, Clinical Science and Molecular Medicine, 1974, 47, 289.

6 Thomas, T H, et al, Clinical Science and Molecular Medicine, 1977, 53, $10 \mathrm{P}$.

- Ashton, M G, et al, British Medical fournal, 1977, 1, 1134.

${ }^{8}$ Smith, N J, et al, British Medical fournal, 1977, 2, 804.

\title{
Haemophilia A home therapy in the United Kingdom 1975-6
}

\author{
PETER JONES， MAUREEN FEARNS， CHARLES FORBES， JOHN STUART
}

British Medical fournal, 1978, 1, 1447-1450

\section{Summary and conclusions}

Data on home treatment for patients with haemophilia A (factor VIII deficient haemophilia) were compiled for 1975 and 1976 from questionnaires answered by directors of haemophilia centres throughout the United Kingdom. There were 48 haemophilia centres in 1975 and 71 in 1976. The number of patients on or in training for home treatment increased from 267 to 488 in the two years, and a further 241 haemophiliacs were considered suitable for home therapy by the end of 1976. Apart from a small (but increasing) number of haemophiliacs on prophylactic treatment, most patients were on low-dose (250-500 units) on-demand regimens, using a mean of 20000

\footnotetext{
Newcastle Haemophilia Centre, Royal Victoria Infirmary, Newcastle upon Tyne

PETER JONES, MD, MRCP, consultant paediatrician

MAUREEN FEARNS, SRN, sister

Royal Infirmary, Glasgow

CHARLES FORBES, MD, FRCP, consultant physician

Queen Elizabeth Hospital, Birmingham

JOHN STUART, MD, FRCP, consultant haematologist
}

factor VIII units per patient per year in 1976. An estimated $55 \%$ of the blood product used for home therapy in the UK in 1976 was imported from commercial sources.

Despite the fact that the numbers of patients on home treatment have increased, so that about $60 \%$ of the potential population were receiving or being considered for home treatment in 1976, inadequacies in the service still remain. In some centres follow-up is clearly inadequate; about $15 \%$ of patients still rely on cryoprecipitate; and too little money has been invested in making the NHS self-sufficient in factor VIII production.

\section{Introduction}

The practice of training haemophilic patients to treat themselves at home with intravenous injections of factor VIII dates from the more widespread introduction of concentrated blood fractions in the mid 1960s. Before the discovery of cryoprecipitate in $1964^{1}$ these fractions were in short supply and usually reserved for surgery and the treatment of major complications in hospital, most haemophiliacs receiving fresh frozen plasma for the routine management of haemorrhage. The introduction of cryoprecipitate allowed outpatient treatment for all but the most severe bleeding episodes, and it was a logical step to train experienced patients to set up their own transfusions under supervision. Some haemophiliacs began to store cryoprecipitate 
at home to avoid the inevitable delays experienced with hospitalbased treatment, and they either administered their own injections or received them from their family doctors. ${ }^{2}$

There are, however, many difficulties in using cryoprecipitate for home treatment-it must be stored deep frozen, several packs must be thawed and pooled to make up a recommended dose, and the precise amount of factor VIII per pack is unknown. Therefore it was not until lyophilised (freeze-dried) concentrates were more freely available that large-scale home treatment programmes became possible. Within the United Kingdom the first commercially prepared factor VIII lyophilised concentrates were licensed by the Department of Health and Social Security in November 1973, and by 1975 home treatment programmes were being run by several haemophilia centres. This report summarises the growth and development of haemophilia home treatment in the United Kingdom in 1975 and 1976.

\section{Method}

In January $1976^{3}$ and January 1977 questionnaires were sent to the directors of the United Kingdom haemophilia centres. There were 48 centres in 1976 and 71 in 1977. All made returns, the last of the completed questionnaires for 1976 being returned in October 1977. The 1976 returns contained the answers to an inquiry on the directors' recommendations for starting and running a home treatment programme as well as questions relating to the previous year's experience. These questions dealt with the numbers of patients on home treatment or in training and the numbers considered suitable for future training. The directors were asked for details of the blood products prescribed and their recommended doses and for the mean annual amount of factor VIII used by their patients on home treatment (excluding surgery and other inpatient requirements). They were also asked about patients receiving prophylactic treatments and haemophiliacs with antibodies to factor VIII who were on home therapy.

Not all directors replied to every question, and the results were adjusted accordingly. Some patients attended more than one centre

TABLE I-Comparative data on numbers of centres and numbers of patients in 1975 and 1976

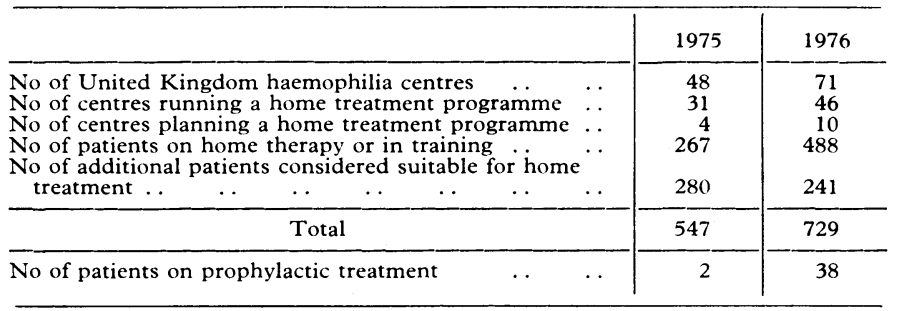

for treatment-for example, boys attending boarding school in a different region from their homes. The number of these patients was listed in each return and the figures adjusted after cross checking.

\section{Results}

Number of patients-All th naires in both 1976 and 1977. Thirty-nine of the 48 centres had started home treatment by the end of 1975 and 46 of the 71 centres by the end of 1976. Over the two years the number of haemophiliacs ono home treatment or in training rose from 267 to 488 . Two patientsర were on prophylactic therapy in 1975 and 38 in 1976 (table I). In both surveys the directors were asked to assess the additional numbers of haemophiliacs attending their centres who they considered suitable for home therapy; 280 additional patients were reported for 1975 and 241 o for 1976. Thus at the end of 1976 there were 729 haemophiliacs $\vec{\circ}$ either on or awaiting home treatment in the United Kingdom.

Blood products prescribed - Seven factor VIII products were available $\vec{\omega}$ in 1975 and nine in 1976 (table II). Despite increased supplies of lyophilised concentrate 15 centres were still issuing cryoprecipitate for home treatment in 1976. The increased output of Lister Institute. concentrate, prepared at the Elstree fractionation plant from volunteer. donor plasma collected in England, Wales, and Northern Ireland, was 3 reflected in the returns. Thirty-five centre directors, representing $425 \mathrm{~N}$ patients, gave sufficient information on blood product use to allow the $\vec{A}$ prescription of NHS source products and that of imported commercial products to be compared in 1976 (table III). Forty-five per cent of the blood product used for home treatment in these centres came from 을 NHS sources $(15 \%$ cryoprecipitate; $30 \%$ lyophilised concentrate), $\omega$ and $55 \%$ came from abroad. An estimated 7874750 units of factor VIII were used by patients in these 35 centres during the year, 4395370 units being from commercial sources. Cryoprecipitate unitage was estimated on mean of 70 factor VIII units per pack. Fourteen centres, representing 112 patients, used only one product $\infty$ for home treatment.

Factor VIII dosage-All directors calculated their recommended doses to the nearest phial or pack so that blood product was never wasted. Of the 46 directors replying to the question, 33 based their recommendations on clinical response, 11 on both clinical response 0 and assayed in-vivo factor VIII response, and two on assayed factor $\varrho$ VIII response only. The average phial of concentrate contains $250 \stackrel{\vec{F}}{\vec{B}}$ factor VIII units (range 210-310 units). The 33 directors who based their dosage on clinical response recommended that one or two phials be given on demand-that is, when the patient recognised that a bleed had started. Seven prescribed one phial as routine, eight two phials, and 13 one phial as routine with two phials for major joint bleeds. Five directors recommended one phial as routine for children and two for adults; apart from this distinction only five other directors 0 mentioned body weight as a factor to be taken into account when calculating dosage for home treatment. Most directors issued only enough blood product for one to two months' treatment at a time. 3 Both the practice of measuring in-vivo factor VIII activity response . 
TABLE V-Criteria for home therapy (51 centres)

\begin{tabular}{|c|c|c|}
\hline \multicolumn{2}{|l|}{ Criteria } & \multirow{2}{*}{$\frac{\text { No of centre }}{35}$} \\
\hline $\begin{array}{l}\text { Severe clinical disease with frequent bleeds } \\
\text { Sufficient intelligence to follow instructions, rec } \\
\text { treatment criteria, maintain records, and co-o }\end{array}$ & $\underset{\text { perate }}{\cdots}$ & \\
\hline $\begin{array}{lllll}\text { with centre } & \ldots & \ldots & \ldots & \ldots\end{array}$ & ... & 31 \\
\hline Good veins $\ldots$ & .. & 23 \\
\hline Psychological stability, reliability, and good sens & se & 20 \\
\hline Ability to perform atraumatic venepuncture & . & 17 \\
\hline Motivation and willingness to give injections & .. & 16 \\
\hline Distance from centre $\quad \ldots$ & . & 16 \\
\hline Absence of clotting factor antibodies & . & 14 \\
\hline Good home environment .. & . & 15 \\
\hline Age $\quad \because \quad \therefore \quad \ldots \quad \ldots$ & . & 7 \\
\hline Prevention of joint damage.. & . & 3 \\
\hline Agreement of family doctor $\quad \ldots \quad \ldots$ & $\therefore$ & 3 \\
\hline Absence of allergic reactions to blood products & $\therefore$ & 1 \\
\hline Absence of hepatitis B antigen $\quad . . \quad \ldots$ & $\therefore$ & $i$ \\
\hline Absence of alcoholism or drug abuse & .. & 1 \\
\hline
\end{tabular}

and the rise in activity recommended for a particular bleeding episode varied from centre to centre. The times of blood sampling for in-vivo response were reported as 10 minutes ( 8 centres), 15 minutes (1) 20 minutes (3), and 30 minutes (1). Recommended VIII activity varied between $20 \%$ for "minor" bleeds and $40 \%$ for "major" bleeds and haemarthroses.

Factor VIII used-Twenty-eight directors reported the mean number of factor VIII units used exclusively for home therapy per patient in 1976 (table IV). The overall mean was 19920 (with a range of 3505-55 390) factor VIII units per patient per year. In 1975 the mean had been calculated at 25000 factor VIII units per patient per year (range $3000-80000$ ), but it was evident that this figure was skewed, a relatively large number of the most severely affected patients being started on home treatment first. ${ }^{4}$ The figures for the three centres with the longest established programmes in 1975 (Newcastle, Oxford, and the Royal Free Hospital) showed lower mean values: 18796,17000 , and 10000 factor VIII units respectively in 1975, and 20 793, 17000 (approximately), and 15300 factor VIII units in 1976.

Patients with antibodies to factor VIII-Four patients with factor VIII antibodies were reported to be on home treatment in 1976. In two cases the antibody titre fell despite continued treatment with factor VIII concentrates. One patient received factor IX preparations only, and one both factor VIII and factor IX. In both cases home treatment was discontinued because of rising antibody titres.

Criteria for home treatment-Fifty-one directors listed their criteria for starting a haemophiliac on home treatment (table V). Most (59\%) considered that a telephone within the patient's home was mandatory, and all agreed that immediate access to a telephone was essential Four directors $(8 \%$ ) asked their patients to contact the centre before injecting themselves. Thirteen $(27 \%$ ) recommended an occasional home visit by a member of the centre staff as a requirement for home treatment.

Follow up-The directors were asked to specify their procedures for following up haemophiliacs on home treatment. Forty replied to this section of the 1976 questionnaire and on the basis of their replies they were divided into four groups. Six centres performed regular follow-up at least once a year, including full physical examination with musculoskeletal survey, radiology, and blood profile (at least a blood count, estimation of factor VIII activity, antibody screen, liver function tests, and screening test for hepatitis B antigen and antibody). They had access to specialists in orthopaedics, physical and social medicine and dentistry. Fourteen centres performed regular follow-up at least once a year which included at least a physical examination and measurement of joint ranges and blood count; one of these centres was for children only and saw adults only on an ad hoc basis. Sixteen centres merely checked the home transfusion records when patients presented for further supplies of blood product, and performed a blood count. The last group, of four centres, only checked the home treatment records. In all centres a blood count included estimation of haemoglobin and other red cell indices, white cell and platelet counts, and examination of a blood film.

\section{Discussion}

The two surveys reported show the recent rise in the use of home treatment for patients with haemophilia A in the United Kingdom. In 19741589 severely affected (factor VIII <2\% haemophiliacs of all ages were known to the haemophilia centre directors. ${ }^{5}$ It may be assumed that about $25^{\circ}$ of these patients were unsuitable for home treatment because of age, infrequency of haemorrhage, presence of factor VIII antibody, or inability to fulfil other criteria. ${ }^{4}$ The number of haemophiliacs (729) either on home treatment or reported as being suitable for it at the end of 1976 therefore represents about $60^{\circ} \%$ of the potential total.

The 1976 figures for those patients either on home treatment or in training indicated an annual factor VIII requirement of 9.76 million units $(488 \times 20000)$, with a potential annual requirement of 23.8 million units exclusively for home treatment. This is more than half the estimated total national requirement of 40 million units of factor VIII reported by Biggs, ${ }^{5}$ the remaining blood product being required to treat patients not on home treatment and to cover surgery and the management of complications, including the development of factor VIII antibodies.

Apart from the numbers of patients concerned, there are four variables that govern the demand for factor VIII for home treatment-the assessment of effective dosage, the use of prophylaxis, the adequacy of supply, and the risk of long-term side effects. Our results show that in the UK severely affected haemophiliacs are trained to treat themselves with comparatively low doses of factor VIII, the mean of 20000 units per patient per year being about half that reported from some other countries. ${ }^{+}$This difference in dosage is very important in relation to the cost of home treatment, and there is an urgent need for comparative trials to determine whether it is of long-term clinical significance.

Although the rise in the use of prophylaxis reported in 1975 and 1976 is expected to be sustained in 1977 it is too early to calculate accurately the long-term effect of this type of treatment on blood product demand. In most cases reported prophylaxis was prescribed temporarily for a specific reason, usually to halt recurrent haemorrhage into a joint already damaged by haemophilic arthropathy, a return to on-demand treatment being indicated once the joint is quiescent with good function. Use of this limited prophylaxis was responsible for the $10 \%$ rise in factor VIII consumption in the Newcastle centre in 1976. Estimates of increased factor VIII demand for prophylaxis vary widely, ${ }^{6-10}$ and, as with on-demand treatment, much of this variation seems to depend on the therapeutic aim, total suppression of haemorrhage demanding very high factor VIII consumption.

Home treatment for many of the haemophiliacs in the United Kingdom would have been impossible without recourse to factor VIII concentrates prepared by pharmaceutical companies. About $55 \%$ of the blood product used for home treatment in 1976 was imported, importation being necessary because of continued shortfall in the production of NHS concentrate from the voluntary donor system of the Blood Transfusion Service. The cost of producing NHS concentrate has not been disclosed, but at a minimum commercial price of $8 p$ per unit the potential cost of home treatment in the United Kingdom will be about $£ 2 \mathrm{~m}$ a year. In 1976 the Department of Health announced that the UK requirement for factor VIII concentrate would be met from NHS sources by mid-1977 after the grant of an additional $£ 0.5 \mathrm{~m}$ to the Blood Transfusion Service. ${ }^{11}$ This target has clearly not been met, nor could it have been in the absence of the necessary financial aid to increase fractionation capacity. It seems poor economic policy to invest only $£ 0.5 \mathrm{~m}$ in a service which produces a very satisfactory factor VIII product and which could be self-sufficient, while continuing to pay $£ 1 \mathrm{~m}$ to $£ 2 \mathrm{~m}$ a year on blood from paid donors in other countries. The difficulty of implementing home treatment in some areas is reflected in the continued use of cryoprecipitate, which, despite its disadvantages, remained the only product available for $15 \%$ of haemophiliacs in 1976 .

Both the need to carefully monitor the use of blood products and the need for continual surveillance of patients because of the possible long-term side effects of intensive transfusion demand adequate follow-up procedures. Perhaps the most disturbing aspect of the 1976 inquiry was the lack of adequate 
follow-up in some centres. The contemporary management of haemophilia is time-consuming and if it is to be effective it demands the integrated (usually part-time) services of many medical and paramedical workers. Many haemophilia centres still lack the staff and facilities required, despite the obligation on the Health Authorities to provide these, as set out in the Department of Health and Social Security Health circular HC(76)4.

We thank the haemophilia centre directors and their staff for their kind participation in the preparation of this report. The authors are members of the working party on home therapy of the UK haemophilia centre directors.

Requests for reprints should be addressed to Dr P Jones, Newcastle Haemophilia Reference Centre, Department of Haematology, Royal Victoria Infirmary, Newcastle upon Tyne NE1 4LP.

\section{References}

${ }^{1}$ Pool, J, Hershgold, E, and Pappenhagen, A, Nature, 1964, 203, 312.

${ }^{2}$ Le Quesne, B, et al, Lancet, 1974, 2, 507.

3 Jones, P, Scandinavian fournal of Haematology, 1977, suppl No 31, p 19.

Jones, P, Proceedings of 3rd European Congress of the World Federation of 0 Hemophilia. London, 1976. In press.

5 Biggs, R, and Spooner, R, British Fournal of Haematology, 1977, 35, 487. ${ }^{6}$ Nilsson, I M, Hedner, U, and Ahlberg, A, Acta Paediatrica Scandinavica, $\varrho$ 1976, 65, 129.

${ }^{7}$ Kasper, C K, Dietrich, S L, and Rapaport, S I, Archives of Internal Medicine, 1970, 125, 1004.

${ }^{8}$ Ramsay, D M, and Parker, A C, Fournal of Clinical Pathology, 1973, 25, 243.

9 Aronstam, A, et al, British fournal of Haematology, 1976, 33, 81.

10 Carter, F, et al, British Medical fournal, 1976, 2, 465.

11 Hansard, House of Commons, 26 February, 1975, vol 884 col 392.

\title{
Circulating immune complexes in pre-eclampsia
}

\author{
G M STIRRAT, C W G REDMAN, R J LEVINSKY
}

British Medical fournal, 1978, 1, 1450-1451

\section{Summary and conclusions}

Sixteen patients with severe pre-eclampsia had more IgG-containing and C1q-binding circulating soluble immune complexes than did 16 matched women with normal pregnancies. The clinical features of preeclampsia may be explained by damage due to such complexes, although raised complex levels were also present in a few women with normal pregnancies.

As immune complexes are so heterogenous in terms of the type of antigen, class and subclass of immunoglobulin, size, and complement-binding capacity, further investigations are needed to determine their role in normal and pre-eclamptic pregnancies.

\section{Introduction}

Soluble immune complexes have been reported in the sera of women in normal pregnancy, ${ }^{1}$ and these may arise from a physiological immunological reaction between mother and child. Immune complexes can, however, cause a wide range of diseases, depending on, for example, the amount and type of complex. Many of the features of pre-eclampsia might be explained by excess or qualitatively abnormal circulating soluble immune complexes. ${ }^{2}$ We have therefore measured IgG-containing and Clq-binding complexes in the sera of women with severe pre-eclampsia and closely matched women with normal pregnancies.

\section{Patients and methods}

Sixteen pregnant women with severe pre-eclampsia were studied at gestational ages ranging from 28 to $\mathbf{4 0}$ weeks. The criteria for diagnosis were: (a) an increase of $30 \mathrm{~mm} \mathrm{Hg}$ or more in the systolic blood

John Radcliffe Hospital, Headington, Oxford

G M STIRRAT, MD, MRCOG, clinical reader

C W G REDMAN, MRCP, lecturer in obstetric medicine

Department of Immunology, Institute of Child Health, London WCIN 1EH

R J LEVINSKY, BSC, MRCP, research fellow in immunology

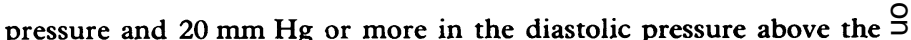
first reading recorded in the pregnancy (always before 20 weeks); and $\omega$ (b) proteinuria of over $200 \mathrm{mg} / \mathrm{l}$ in three midstream uninfected urine samples or more than $1 \mathrm{~g}$ per 24 hour urine collection.

Sixteen women with normal pregnancies (controls) were selected $\vec{D}$ who matched the patients for gestational age and parity (except for one para 3 control who was matched with a para 4 patient).

Venous blood samples were allowed to clot at room temperature, separated within three hours, and stored in aliquots in liquid nitrogen until analysis. IgG immune complexes were measured by their inhibition of IgG latex agglutination by rabbit IgM anti-human $\mathrm{IgG}^{3}$; agglutination was measured by counting unagglutinated $\frac{\mathrm{D}}{\mathrm{Q}}$ particles on an electronic counter. Clq-binding complexes were $\varrho$ similarly measured using purified human $\mathrm{Clq}$ as the agglutinating $\overrightarrow{\vec{B}}$ reagent. ${ }^{3}$ Samples were analysed blind in duplicate and results were 3 expressed as percentage inhibition of agglutination. In non-pregnant people levels of inhibition are considered significant only if they are $\frac{\overrightarrow{0}}{0}$ $20 \%$ or more. ${ }^{3}$

\section{Results}

All 16 patients with pre-eclampsia had detectable levels of IgG complexes, and inhibition of agglutination was greater than $20 \%$ in 11 cases. Five controls had no detectable IgG complexes and only 우 four had more than $20 \%$ inhibition (see table). Similarly, 13 pre- N eclamptic patients had raised levels of Clq-binding complexes ON compared with four controls. For both types of complexes mean $\frac{D}{O}$ titres were considerably higher in the patients than in the controls $(P<0.005$; Wilcoxon's rank sum test). In the group as a whole the $N$ values of patients and controls overlapped, but most of the pre- N eclamptic patients had higher values than their matched controls at $\frac{\omega}{\sigma}$ each gestation studied.

Two patients who had very low levels of IgG complexes at 32 and 29 weeks' gestation (cases 2 and 3) had much higher levels of Clqbinding complexes $(17.7 \%$ and $33.2 \%$ inhibition respectively).

\section{Discussion}

The cause of pre-eclampsia is unknown. It occurs pre- $O$ dominantly in first pregnancies, and even a first trimester $ᄋ$ miscarriage is protective. ${ }^{4}$ This suggests that immunisation to응 fetal antigens may stimulate the maturation of maternal immune reactions that are necessary to prevent fetal allograft rejection. Pre-eclampsia may be caused by partial failure of these protective mechanisms.

Higher levels of circulating soluble immune complexes may not only be a marker of this relative failure but may also cause 